\title{
Electronic Transport in the Biopigment Sepia
}

\section{Melanin}

\author{
Reali, Manuel'; Gouda, Abdelaziz 1'; Bellemare, Jonathan'; Ménard, David'; \\ Nunzi,Jean-MicheR,3; Soavi, Francesca4; Santato, Clara ${ }^{1 *}$ \\ 1Department of Engineering Physics, Polytechnique Montreal, Montreal, H3C 3A7, \\ Canada \\ 2Department of Physics, Engineering Physics \& Astronomy, Queen's University, \\ Kingston, K7L 3N6, Canada \\ ${ }^{3}$ Department of Chemistry, Queen's University, Kingston, K7L 3N6, Canada \\ ${ }^{4}$ Department of Chemistry "Giacomo Ciamician", Alma Mater Studiorum Università di \\ Bologna, Bologna, 40126, Italy
}




\author{
KEYWORDS. Sepia melanin, electronic transport, reversible resistive switching, bio- \\ sourced materials, sustainable (green) organic electronics
}

\begin{abstract}
Eumelanin is the most common form of the pigment melanin in the human body, with diverse functions including photoprotection, antioxidant behavior, metal chelation, and free radical scavenging. Melanin also plays a role in melanoma skin cancer and Parkinson's disease. Sepia Melanin is a natural eumelanin extracted from the ink sac of cuttlefish.
\end{abstract}

Eumelanin is an ideal candidate to eco-design technologies based on abundant, biosourced and biodegradable organic electronic materials, to alleviate the environmental footprint of the electronics sector.

Herein, the focus is on the reversible electrical resistive switching in dry and wet Sepia eumelanin pellets, pointing to the possibility of predominant electronic transport a conditio sine qua non to develop melanin-based electronic devices. These findings shed light on 
the possibility to describe the transport physics of dry eumelanin by the amorphous semiconductor model. Results are of tremendous importance for the development of sustainable organic electronics.

\section{Introduction}

The rapidly growing demand for consumer electronics, one of the most ubiquitous technologies, has led to unsustainable amounts of waste of electrical \& electronic equipment (WEEE). On a global scale, WEEE amounts approximately to 45 megatons per year. ${ }^{1}$ WEEE contains hazardous substances that pose health and environmental concerns. ${ }^{1}$ Refurbishment, recycling and recovery in conventional (inorganic) electronics are viable routes to limit the environmental footprint of consumer electronics. ${ }^{2}$ In parallel, the eco-design of electronic devices based on the use of abundant organic bio-sourced materials that are potentially biodegradable, a field that we call organic green (sustainable) electronics, is of the utmost importance, e.g. for the development of low human- and eco-toxicity technologies for the Internet of Things. 
In the melanin biopigment family, eumelanin is a brown-black subgroup found in the human body, other mammals, birds, reptiles, amphibians and fishes as well as in lower invertebrates, such as cuttlefish and insects. ${ }^{3}$ In humans, aside from photo-protection, eumelanin is involved in a variety of processes, e.g. the accumulation and release of metal cations in the body. ${ }^{4,5}$ It is noteworthy that the interactions between iron and neuromelanin, a pigment made of eumelanin and red-yellow pheomelanin, present in the brain of humans and primates, have been related to Parkinson's disease. ${ }^{6}$ The remarkable adhesion of melanin-like materials on surfaces is the underpinning for emerging applications in the biomedical and water treatment fields. ${ }^{7-9}$ Sepia melanin is a natural eumelanin extracted from the ink sac of cuttlefish (Sepia officinalis). ${ }^{10}$

Eumelanin is an ideal bio-sourced candidate for the exploration of the potential of sustainable organic electronics. Indeed, its molecular structure features electronic conjugation (alternance of single and double bonds), which is a peculiar structural feature of organic semiconductors. ${ }^{11}$ At the same time, beyond its ubiquity among flora and fauna, eumelanin is biocompatible, edible and potentially biodegradable. ${ }^{7,12}$ Considering the presence of redox active quinone groups in the molecular structure, ${ }^{7}$ electronics powered 
by eumelanin-based electrochemical energy storage devices can also be conceived. Indeed, the redox activity of eumelanin has recently enabled a number of technologies, such as melanin-based biological electrodes as well as flexible and light-assisted (micro)supercapacitors. ${ }^{13,14}$

Eumelanin is made up of two building blocks, 5,6-dihydroxyindole (DHI) and 5,6dihydroxyindole-2-carboxylic acid (DHICA), coexisting in different redox states. Hydroquinone $(\mathrm{H} 2 \mathrm{Q})$ is the reduced state, semiquinone $(\mathrm{SQ})$ the intermediate redox state and quinone $(\mathrm{Q})$ the oxidized state, with its tautomer quinone imine (QI) (Scheme 1). The presence of water shifts towards the right the comproportionation equilibrium where $\mathrm{H} 2 \mathrm{Q}$ and $Q$ react to give $S Q$ extrinsic radicals and protons. The latter are the mobile electronic charge carriers in eumelanin ${ }^{15}$ (Scheme 2).

$\mathrm{DHI}$ and DHICA have several polymerization sites making eumelanin a chemically heterogeneous macromolecule. ${ }^{16,17}$ This heterogeneity explains the short-range order in the material that can therefore be defined, to a certain extent, as amorphous. Nevertheless, self-assembled $\pi-\pi$ stacked supramolecular structures with a 
characteristic inter-plane spacing of $\sim 3.7 \AA$ have been observed both with natural and synthetic eumelanin. ${ }^{18}$

In the 1970s, McGinness et al. reported on the reversible electrical resistive switching from a high (OFF) to a low (ON) resistive state observable beyond a threshold voltage $\left(V_{T}\right)$ for hydrated eumelanin pressed pellets. ${ }^{19}$ At that time, resistive switching had been experimentally observed in amorphous $\mathrm{Si}$, amorphous $\mathrm{Ge}$ and chalcogenide glasses (e.g. amorphous Te and amorphous Se) ${ }^{20}$ As a result, the resistive switching behavior shown by eumelanin pellets was interpreted within the Mott-Davis amorphous semiconductor model (ASM). ${ }^{19,21}$ This model also provided an explanation for the broadband UV-Vis absorption of the biopigment. ${ }^{22} \mathrm{~A}$ crucial element in the work of McGinness et al. was the observation that only hydrated eumelanin pellets exhibited electrical resistive switching, i.e. dry pellets did not. The lack of switching of dry pellets was explained by the modified dielectric constant theory, which posits that the adsorbed water increases the dielectric constant of the material, lowering the activation energy for charge transfer processes. ${ }^{23}$ Recent publications on the electrical response of hydrated eumelanin ${ }^{24-26}$ questioned the 
ASM and proposed the mixed ionic-electronic conductor model (Scheme 2 and Table 1). ${ }^{27-29}$

Here, we report on the observation of predominant electronic transport in dry pellets of natural Sepia melanin, processed at room temperature. Pellets exhibit room temperature conductivity of about $10^{-3} \mathrm{~S} \cdot \mathrm{cm}^{-1}$, after switching. Our observation has profound implications for the development of the field of sustainable organic electronics. Indeed, the observation of pure electronic transport paves the way towards the development of Sepia melanin-based transistors and memory devices. Further, long-term implications are also foreseen in the medical and biomedical fields.

\section{Experimental Section}

\section{Sepia melanin extraction}

Sepia melanin was extracted from the ink sac of the cuttlefish Sepia officinalis. First 100 $\mathrm{g}$ of ink were suspended in $200 \mathrm{~mL}$ of $\mathrm{HCl} 2 \mathrm{M}$ and stirred for $24 \mathrm{hr}$ at $10{ }^{\circ} \mathrm{C}$. The suspension was centrifuged in a planetary mixer (THINKY ARM-310) and washed several times with $0.5 \mathrm{M} \mathrm{HCl}$, ethanol, DI water, ethyl acetate and a buffer solution $(0.95 \mathrm{v} / \mathrm{v}$ of 
monobasic sodium phosphate $200 \mathrm{mM}, 40.5 \mathrm{v} / \mathrm{v}$ of dibasic sodium phosphate $200 \mathrm{mM}$, $50 \mathrm{v} / \mathrm{v}$ of DI water). For each step, the suspension was weighed, and the $\mathrm{pH}$ measured. Centrifugation was performed at $10000 \mathrm{rpm}$ at $5{ }^{\circ} \mathrm{C}(25 \mathrm{~min}$ for DI water and buffer solution, $15 \mathrm{~min}$ for $\mathrm{HCl} 0.5 \mathrm{M}$, ethanol and ethyl acetate). After stirring, the centrifugate was sequentially washed with $\mathrm{HCl} 0.5 \mathrm{M}$, DI water, the buffer, and ethanol, and centrifuged at each washing step. Subsequently, the suspension was washed again with DI water and centrifuged three times. The final product was lyophilized for $24 \mathrm{hr}$ to remove solvents. A fine black powder was finally obtained.

\section{Fabrication of Wet Pellets}

The Sepia melanin powder was ground to remove large agglomerates and subsequently weighed (analytical balance, accuracy $10^{-5} \mathrm{~g}$ ). For the hydrated pellets, we measured the weight percent gained by the pellets after $24 \mathrm{hr}$ hydration (\% wt), at different percentages of relative humidity in the atmosphere (Table S1). The percentage of weight gained after hydration was calculated using the following relation: $\% w t=\frac{W_{2}-W_{1}}{W_{1}} \times 100$, 
where $W_{2}$ and $W_{1}$ are the weight after and before hydration respectively. The hydration was performed in a Cole-Parmer Mini Humidity Chamber (03323-14) equipped with an automatic humidity controller and an ultrasonic humidification system (control humidity levels from $20 \%$ to $90 \%$ with programmable set points).

A cylindrical stainless-steel coin cell (inner diameter $15.5 \mathrm{~mm}$, outer diameter $19.5 \mathrm{~mm}$, thickness prior pressing $2.6 \mathrm{~mm}$ ) was used as protective gasket to assemble and seal the pellet. The coin cell was assembled as follows. A pre-cleaned $99 \%$ purity $\mathrm{Cu}$ foil (dimensions of the inner diameter of the coin cell, $0.1 \mathrm{~mm}$ thickness), from Goodfellow Cambridge Limited, was placed inside the coin cell and the hydrated powder gently transferred on the $\mathrm{Cu}$ surface. The top contact was then made by placing the top stainless-steel disk $(0.1 \mathrm{~mm}$ thickness, SUS316L from SteelJIS) on the powder and the sandwich structure sealed with the upper cap (Scheme 3B and C). The coin cell was then manually pressed into $2 \mathrm{~mm}$ thick pellets by applying a pressure of 3 tons for $60 \mathrm{~s}$ by means of a manual hydraulic press machine (Specac's Atlas Series).

Fabrication of Dry Pellets 
Sepia melanin powder was ground to remove large agglomerates and transferred with the coin cell and the pre-cleaned contacts to an argon glovebox $\left(\mathrm{H}_{2} \mathrm{O}<0.1 \mathrm{ppm}\right.$, equipped with a manual hydraulic press machine where it was kept for $24 \mathrm{hr}$ prior to pressing it into pellets. The powder was weighed (analytical balance, accuracy $10^{-5} \mathrm{~g}$ ) and the coin cell assembled following the same fabrication procedure as the hydrated samples. The structure was then pressed into $2 \mathrm{~mm}$ thick pellets ( 3 tons for $60 \mathrm{~s}$ ).

\section{Electrical}

The pellets were clamped to ensure the stability of the contacts during the electrical measurements. Then, they were connected to an amperometer at the terminals of which the current was recorded. The pellets were modeled with an electrical resistance $\left(R_{P}=R_{e u}+R_{c}\right.$ where $R_{e u}$ and $R_{c}$ are the intrinsic eumelanin resistance and the contact resistance, respectively) in series with the internal resistance of the voltage source $R_{S}$. At the threshold voltage $\left(V_{T}\right), R_{P}$ can be calculated as $R_{P}=\frac{V}{I}-R_{S}$, where $V$ is the applied voltage and I the measured current (Scheme 3A). At least five dry and wet Sepia melanin pellets were tested. The reported values of resistances (Figure S2 and Table S3) are average with an error of $\pm 0.5 \%$. The electrical response of the pellets was acquired using a Genesys ${ }^{\mathrm{TM}}$ Lambda-TDK 600-2.6 DC power supply (output 
voltage $600 \mathrm{~V}$, maximum output current 2.6 A). LabView I-V Software was used for data recording and programmed to limit the current of the power supply (output current) to the maximum value supported by the resistor (about $150 \mathrm{~mA}$, Scheme 3A). The room temperature electrical conductivity of the pellets can be calculated using the following equation:

$$
\sigma(295 K)=\frac{t}{A \times R_{p}}
$$

where $t$ is the thickness, $A$ is the inner surface area and $R_{p}$ is the resistance of the pellets.

\section{Chemical}

Before and after the electrical tests, the chemical properties of the hydrated and dry pellets were characterized via Raman Spectroscopy and X-ray Photoelectron Spectroscopy (XPS). For the former, we used a Renishaw InVia Raman spectrometer $\left(\lambda=514 \mathrm{~nm}, 100 \mathrm{~nm} \mathrm{~s}^{-1}\right.$ scanning rate, $5 \%$ of the total power to prevent overheating effects). XPS was performed with a VG ESCALAB 3 MKII apparatus (source Mg Ka, power $300 \mathrm{~W}$ [15 kV, $20 \mathrm{~mA}$ ], pressure in analysis chamber ca. $10^{-9}$ Torr, electron take 


\begin{abstract}
off angle $0 \mathrm{deg}$, analyzed depth less than $10 \mathrm{~nm}$, detection limit $0.1 \%$ at). Survey scans and high-resolution scans were carried out with $1 \mathrm{eV}$ and $0.05 \mathrm{eV}$ energy steps. Background subtraction was performed via the Shirley method and charge correction with respect to $\mathrm{C}_{1 \mathrm{~s}}$ set at $284.7 \mathrm{eV}$.
\end{abstract}

\title{
Electrochemical Impedance Spectroscopy
}

EIS measurements were performed at ambient conditions with a BioLogic bipotentiostat (SP-300). Data were acquired at open circuit potentials (OCP), within the frequency range $3 \mathrm{MHz}-0.1 \mathrm{~Hz}, 20$ points per decade, $10 \mathrm{mV}$ oscillation amplitude.

Scanning Electron Microscopy (SEM) and Energy-Dispersive X-ray (EDX)

A JEOL JSM7600F microscope was used. EDX was carried out using the same microscope with Aztec (Oxford) software, detector x-Max (80 mm², Oxford), at $5 \mathrm{kV}$. 


\section{Results}

\section{Electrical response of Sepia melanin pellets}

The current-voltage characteristics of dry and wet Sepia melanin pellets, placed between $\mathrm{Cu}$ and stainless-steel electrodes, show that for dry pellets, during the first current-voltage scan, the current monotonically increases with the increase of the voltage up to the threshold voltage $V_{T}$, where the electrical resistive switching takes place (Figure 1A). The resistance across the pellets in the ON and OFF states is about $100 \Omega$ and 550 $\Omega$ (the ON resistance, $R_{O N}$, is calculated at the maximum value of the current after switching, whereas the OFF resistance, $R_{\text {OFF }}$, is calculated at $\mathrm{V}_{\mathrm{T}}$ right before switching (see experimental section and Table S2)). For an ON resistance of $100 \Omega$, dry pellets feature a room temperature electrical conductivity of about $1 \times 10^{-3} \mathrm{~S} \cdot \mathrm{cm}^{-1}$, a value falling within the range of electrical conductivity of semiconductors.

From the second current-voltage cycle, the electrical response is significantly different from that of the first. Indeed, the response from the second cycle fits a current-voltage power law of the form $I \propto V^{a}$, with values of $a$ close to or higher than unity (Figures S1 A and C and Table S3). The electrical response of dry pellets features the same current-voltage power law at different voltage scan rates, after the first scan (Figure 1C). The independence of the electrical response on the 
voltage scan rate suggests predominant electronic conduction in the dry pellets. Values of $a \geq 1$ in the power law indicate that, under the effect of an electric field, electronic space charge layers, broadly defined as an excess of electrons or holes over a region of space, form at the Sepia melanin/metal interface. Amorphous materials, such as eumelanin, are prone to host space charge layers due to the presence of structural defects acting as charge carrier traps. ${ }^{30,31}$ To validate this hypothesis, we converted our I-V curves into $\log (\mathrm{J})-\log (\mathrm{V})$ plots, J being the current density, namely the total current per unit area. Our $\log (\mathrm{J})-\log (\mathrm{V})$ plot shows the change of slope between the Ohmic regime and the Space Charge Limited Current (SCLC) regime (Figure S1 E, red and blue solid lines with slope of $0.93 \pm 0.06$ and $2.09 \pm 0.02$ respectively). This change of slope can be attributed to the flow of space charge currents in the presence of a distribution of shallow trap states in organic semiconductors. ${ }^{32}$

Wet Sepia melanin pellets also feature electrical resistive switching (Figures 1B, S1 B and D). No considerable difference is observable between the first and the following scans apart from $V_{T}$ (Figure S1 D). The scan rate has a dramatic effect on the currentvoltage characteristics of wet pellets. At $2000 \mathrm{mVs}^{-1}$ the current starts to increase at about $12 \mathrm{~V}$ to reach a maximum at about $40 \mathrm{~V}$. This maximum likely results from the increase of the electronic component of the current and the (exponential) decrease of the ionic component, at this rate. In addition, the electrical response for the whole interval $0 \mathrm{~V}$ to $180 \mathrm{~V}$ shows that, beyond $2000 \mathrm{mVs}^{-1}$, the resistive switching is not observable, and the 
electrical response fits a power law (Figures 1D, S2 and Table S3). From these results, we infer that conduction in wet pellets is predominantly electronic at high voltage scan rates.

After the resistive switching, we followed the evolution of the resistance with time, for dry and wet pellets (Figure S3). Dry and wet pellets have an initial resistance of $130 \Omega$ and $225 \Omega$. The resistance of dry and wet pellets increases up to about $1200 \Omega \mathrm{in} 10 \mathrm{hr}$ (in the first $5 \mathrm{hr}$ the resistance of the dry pellets increases by ca. $27 \Omega \mathrm{hr}^{-1}$ while the resistance of the wet counterparts by ca. $155 \Omega \mathrm{hr}^{-1}$ ). Such an increase of resistance with time points to volatile memory behavior of both dry and wet Sepia melanin pellets, analogous to organic polymer-based memory devices. ${ }^{33}$ To gain insights into the relaxation processes taking place after resistive switching, we measured the current-time characteristics at constant electrical bias. Dry pellets show a plateau-like behavior after an initial increase of the current, most likely attributable to an electric field-induced supramolecular arrangement favorable to electronic transport (Figure 2) $\cdot{ }^{34}$ On the other hand, the transient current characteristics of wet pellets show an exponential decay likely 


\begin{abstract}
due to the formation of ionic double layers, followed by a plateau-like behavior attributable to electronic transport (Figure 2 and Table S4).
\end{abstract}

\title{
Electrochemical Impedance Spectroscopy
}

To shed light on ionic and electronic processes featuring different relaxation times, as well as possible interfacial processes at Sepia melanin/metal interfaces, we performed an Electrochemical Impedance Spectroscopy (EIS) study with frequency ranging from 3 $\mathrm{MHz}$ down to $0.1 \mathrm{~Hz}$. Nyquist plots (imaginary vs real components of the complex impedance) obtained from dry pellets before resistive switching feature one semicircle and a low-frequency diffusion tail (Figure 3A). We associate the high-frequency semicircle with the electronic impedance of the dry pellet and the Sepia melanin/metal electrode interfacial impedance. The low-frequency tail describes electronic diffusion processes in dry Sepia melanin (suggesting the hypothesis that ionic contribution can be excluded). Assuming a simple resistor-capacitor behavior $(\mathrm{RC})$ with a semicircle, a relaxation time of $17.4 \mathrm{~ms}$ is obtained (this is the $1 / \mathrm{f}_{\max }$ value where $\mathrm{f}_{\max }$ is the frequency of the top of the semicircle, about $57.5 \mathrm{~Hz}$ ). On the other hand, Nyquist plots of the wet pellets before 
resistive switching exhibit three semicircles with relaxation times of $1.33 \mu \mathrm{s}, 0.31 \mathrm{~ms}, 6.14$

ms (Figure 3C). We propose that the two high-frequency semicircles are attributable to the ionic and electronic impedance in the bulk whereas the low-frequency one is attributable to the charge transfer impedance at the Sepia melanin/metal interfaces. The presence of three semicircles suggests electronic conduction in parallel with ionic conduction. ${ }^{35,36}$ The overall impedance should include the capacitance developing at the interface. Nyquist plots of wet and dry pellets do not show any low-frequency capacitive line. Considering the thickness of the pellets (ca. $2 \mathrm{~mm}$, see experimental section), the capacitance is expected to be insignificant. Before the electrical resistive switching, wet pellets show lower impedance than their dry counterparts. This observation can be rationalized in terms of the comproportionation equilibrium, which induces a high density of SQ extrinsic radicals (mobile charge carriers) and protons in wet pellets with respect to dry ones, and leads to the decrease of ionic and electronic impedance. ${ }^{27}$ After the electrical resistive switching, we observe that dry and wet pellets exhibit only one semicircle in the EIS spectra (Figures $3 B$ and D). The buildup of an electrical double layer under the action of the electric field, expected to be electronic for dry pellets and 
predominantly ionic for their wet counterparts, likely facilitates charge carrier injection in the two types of pellets. We also observe that the real part of the total impedance of wet pellets, after the electrical resistive switching, is higher than that of dry pellets. This observation could be attributed to possible side reactions at the wet Sepia melanin/metal interface that passivate the metal contacts.

To better quantify the impedance changes before and after the electrical resistive switching, for both dry and wet Sepia melanin pellets, we fitted the EIS with a simplified $\mathrm{R}_{\text {tot }} \mathrm{Q}$ circuit where $\mathrm{R}_{\text {tot }}$ is the total resistance in series with the constant phase element Q (Table S5). The total resistance includes the electronic $\left(R_{e}\right)$, ionic $\left(R_{i}\right)$ and interface resistance $\left(R_{i f}\right)$ such that $R_{\text {tot }}=R_{e}+R_{i}+R_{i f}$. The constant phase element is the series combination of the melanin's geometrical capacitance and the capacitance at the Sepia melanin/metal interface that describes any deviation from an ideal capacitive response. The fitting parameters indicate that the value of $\mathrm{Q}$ (the capacitive element) for dry pellets increases from ca. $6.70 \times 10^{-12} \mathrm{~F} . \mathrm{s}^{\mathrm{n}-1}$, before switching to $6.77 \times 10^{-9} \mathrm{~F} . \mathrm{s}^{\mathrm{n}-1}$, after switching (Table S5). This increase in capacitance after switching for the dry pellet confirms the formation of a space charge layer that facilitates the charge carrier injection. Considering that the thickness and the section of the pellet do not change before and after switching, the dielectric constant of melanin increases too after switching.

On the other hand, the total capacitance of the wet pellet decreases from ca. $2.60 \times 10^{-8}$ F.s $\mathrm{s}^{\mathrm{n}-1}$, before switching to $3.80 \times 10^{-10}$ F.s ${ }^{n-1}$, after switching. The aforementioned decrease in capacitance of the dry pellet, is in agreement with the hypothesis of the formation of a passivating layer at the wet Sepia melanin/metal interfaces (Table S5). 


\section{Scanning Electron Microscopy}

To evaluate the possibility of explaining the resistive switching by the formation of metallic conductive filaments bridging the interelectrode distance by electrode dissolution, ${ }^{20,24}$ we investigated the surface of the electrodes after switching by Scanning Electron Microscopy (SEM). SEM images and Energy Dispersive X-ray spectra (EDX) do not show any trace of metallic filaments (Figures S4 and S5). Therefore, we tentatively exclude the formation of metallic filaments as the cause of the resistive switching.

\section{Raman Spectroscopy}

We evaluated the possibility of attributing the resistive switching to a phase transition from amorphous to graphitic carbon by Joule heating. ${ }^{20}$ To this end, we performed Raman spectroscopy surveys of dry and wet Sepia melanin powder samples obtained from pellets before and after resistive switching (Figures S6 A, B, C and Table S6). Raman spectra of dry and wet Sepia melanin do not show any change before and after switching. 
We did not observe any peak or shoulder attributable to ordered $\mathrm{sp} 2$ carbon expected at $2750 \mathrm{~cm}^{-1}\left(2 \mathrm{D}_{2}\right.$ band) and at $2700 \mathrm{~cm}^{-1}\left(2 \mathrm{D}_{1}\right.$ shoulder $){ }^{37}$

\section{$X$-ray Photoemission Spectroscopy and X-ray Diffraction}

In agreement with the results of Raman spectroscopy, the high-resolution C1s X-ray photoemission (XPS) spectra of dry Sepia melanin powders, acquired before and after the current-voltage measurements, do not show evidence of graphitic carbon (Figures S6 D and E, Tables S7 and S8). Typical signatures of graphitic carbon are a $\mathrm{C}_{1 \mathrm{~s}}$ peak at $\mathrm{BE}=291.4 \mathrm{eV}$ and an unusually thin $\mathrm{C}_{1 \mathrm{~s}}$ component at $\mathrm{BE}=284.6 \mathrm{eV}$. The high-resolution $\mathrm{C}_{1 \mathrm{~s}} \mathrm{XPS}$ spectra of dry Sepia melanin powder show a satellite peak at $\mathrm{BE}=291.4 \mathrm{eV}$; this is expected in eumelanin because of the cyclic carbon structure. The $\mathrm{C}_{1 \mathrm{~s}}$ component at $\mathrm{BE}=284.7 \mathrm{eV}$ is broad and not as sharp as expected in graphitic carbon (Figure S6 E).

The concentration of $\mathrm{C}-\mathrm{O}, \mathrm{C}-\mathrm{OH}$, or $\mathrm{C}-\mathrm{O}-\mathrm{C}$ groups $(\mathrm{BE}=286.7 \mathrm{eV})$ is high compared to the other chemical groups observed (Tables S7 and S8). The concentration of C-O groups, compared to $\mathrm{C}-\mathrm{N}$ and $\mathrm{C}=\mathrm{C}$ groups, is also higher than expected. Nevertheless, the $C_{1 s}$ spectra before and after switching of Sepia melanin powders are similar, except 
for a slight increase in $\mathrm{C}-\mathrm{O}$ and decrease in $\mathrm{C}-\mathrm{N}$ after switching. X-ray Diffraction (XRD) patterns of dry Sepia melanin powders before and after the electrical resistive switching do not show any signature of graphitic planes (Figure S7), expected as sharp peaks located at $2 \theta \sim 27^{\circ}$ and $2 \theta \sim 54^{\circ} .38$

\section{Discussion}

We propose a tentative model to explain our results, based on the hypothesis that in dry pellets charge carrier injection takes place at sufficiently high applied voltages causing the formation of space charge layers namely electronic double layers at the Sepia melanin/metal interface (Scheme 4). A further increase of the voltage leads to traps filling 30,31 and to the effective overlap of the tails of the functions describing the density of energy states of the valence and conduction bands, eventually leading to the resistive switching.$^{20}$ In our model, the tails stem from the amorphous nature of the Sepia melanin material. From the second current-voltage scan, dry pellets are in a trap-free regime and the current-voltage characteristics can be described by a power law. ${ }^{20}$ For the wet pellets, space charge layers with ionic nature at the Sepia melanin/metal interfaces would lead to 
the electrical resistive switching (Scheme 4). The mixed protonic-electronic conductor model for the wet pellets is supported by the linearization of the current-voltage characteristics at voltage scan rates higher than $2000 \mathrm{mVs}^{-1}$. Work is in progress to locate the Highest Occupied Molecular Orbital (HOMO) and Lowest Unoccupied Molecular Orbital (LUMO) energy levels of Sepia melanin, by Ultraviolet Photoelectron Spectroscopy (UPS) and Inverse Photoemission Spectroscopy (IPES), with the aim to optimize the charge carrier injection process.

\section{Conclusion}

In conclusion, dry and wet Sepia melanin pellets included between metal electrodes feature reversible electrical resistive switching. Our dry and wet pellets feature a conductivity of about $10^{-3} \mathrm{~S} \cdot \mathrm{cm}^{-1}$ after switching. This value refers to natural eumelanin pellets processed at room temperature. We propose that in dry Sepia melanin pellets, under bias, the effective overlap of the density of energy states of valence and conduction band leads to the electrical resistive switching. Electrical double layers (expected to be electronic in dry pellets and ionic in wet ones) enable charge carrier injection at the Sepia 
melanin/metal interface. This behavior points to the validity of the amorphous semiconductor model to describe the transport physics of dry Sepia melanin. The linearization of the current-voltage characteristics after switching and at different voltage scan rates is a strong indication of predominantly electronic conduction. However, the buildup of ionic double layers at voltage scan rates below $2000 \mathrm{mVs}^{-1}$ and the linearization of the current-voltage characteristics at higher voltage scan rates support the mixed protonic-electronic conduction model to describe the transport physics of wet Sepia melanin pellets, in agreement with the literature.

The observation of predominant electronic transport in Sepia melanin satisfies the conditio sine qua non to start designing and developing Sepia melanin-based devices. Our results are of the highest importance for the development of the field of sustainable organic electronics that aims at alleviating the environmental footprint of the electronics sector using abundant, solution processable, possibly biodegradable electronic materials, including biomolecules extracted from agri-waste and biomass feedstock. 


\section{Supporting Information}

Sequential current-voltage scans and cycles of Sepia melanin pellets (Figures S1 and S2), mapping of resistance as a function of time in unbiased condition for Sepia melanin pellets (Figure S3), SEM and EDX analysis of copper and stainless-steel electrodes

(Figures S4 and S5), Raman spectroscopy and XPS survey of Sepia melanin pellets (Figure S7), XRD patterns of Sepia melanin pellets before and after the resistive switching (Figure S8).

\section{Conflict of interest}

The authors declare no competing financial interest.

\section{Corresponding Author}

${ }^{*}$ Correspondence and requests for materials should be addressed to C.S. (email: clara.santato@polymtl.ca) 


\section{Author Contribution}

C. Santato and M. Reali designed the experiments. M. Reali and A. Gouda fabricated

dry and wet Sepia melanin pellets. J. Bellemare designed the electrical circuit and programmed the software to measure the current-voltage characteristics of Sepia melanin pellets. M. Reali performed the current-voltage and the transient current measurements.

A. Gouda acquired the electrochemical impedance spectroscopy response. C. Santato supervised the experiments. All authors gave their critical contribution to data interpretation. M. Reali and A. Gouda drafted the manuscript. All authors made comments and proofread the manuscript.

\section{Acknowledgments}

Dr S. Elouatik (Raman spectroscopy), Dr J. Lefevbre (XPS), P. Plamondon (SEM and EDX) are gratefully acknowledged for technical support and discussions of the results. A. 
Gouda acknowledges financial support from the Institut de l'Energie Trottier and FQRNT through PhD scholarships. C. Santato acknowledges financial support from NSERC (Discovery grant and Strategic Green Electronics Network: grant number: NETGP 508526-17), FQRNT (Team grant) and MESI-Quebec.

\section{References}

(1) Balde, C. P.; Forti, V.; Gray, V.; Kuehr, R.; Stegmann, P., The Global E-Waste Monitor 2017. Quantities, Flows and Resources.; United Nations University, 2017;pp 1-106.

(2) Meloni, M.; Souchet, F.; Sturges, D. Circular Consumer Electronics: An Initial Exploration. Ellen MacArthur Foundation 2018, pp 1-17.

(3) Prota, G. Melanins, Melanogenesis and Melanocytes: Looking at Their Functional Significance from the Chemist's Viewpoint. Pigment Cell Res. 2000, 13 (4), 283293.

(4) Melanins and Melanosomes, Biosynthesis, Biogenesis, Physiological and Pathological Functions, Patrick A. Riley, J. B., Eds.; Wiley-VCH Weinheim, 
Germany, 2011.

(5) Mostert, A. B.; Rienecker, S. B.; Noble, C.; Hanson, G. R.; Meredith, P., The Photoreactive Free Radical in Eumelanin. Science Advances 2018, 4 (3), 1-6.

(6) Fedorow, H.; Tribl, F.; Halliday, G.; Gerlach, M.; Riederer, P.; Double, K. L., Neuromelanin in Human Dopamine Neurons: Comparison with Peripheral Melanins and Relevance to Parkinson's Disease. Progress in Neurobiology 2005, 75 (2), $109-124$.

(7) Bettinger, C. J.; Bruggeman, J. P.; Misra, A.; Borenstein, J. T.; Langer, R., Biocompatibility of Biodegradable Semiconducting Melanin Films for Nerve Tissue Engineering. Biomaterials 2009, 30(17), 3050-3057.

(8) Liu, Y., Ai, K., Lu, L., Polydopamine and Its Derivative Materials: Synthesis and Promising Applications in Energy, Environmental, and Biomedical Fields. Chemical Reviews 2014, 114, 5057.

(9) Haeshin, L.; Dellatore, S. M.; Miller, W. M.; Messersmith, P. B., Mussel-Inspired Surface Chemistry for Multifunctional Coatings. Science 2007, 318, 426-430.

(10) Lindgren, J.; Uvdal, P.; Sjövall, P.; Nilsson, D. E.; Engdahl, A.; Schultz, B. P.; Thiel, 
V., Molecular Preservation of the Pigment Melanin in Fossil Melanosomes. Nature Communications 2012, 3, 1-7.

(11) Malliaras, G.; Friend, R., An Organic Electronics Primer. Physics Today 2005, 58 (5), 53-58.

(12) Di Mauro, E.; Rho, D.; Santato, C., Biodegradation of Bio-Sourced and Synthetic Organic Electronic Materials: Towards Green Organic Electronics. Submitted, 2020.

(13) Xu, R.; Gouda, A.; Caso, M. F.; Soavi, F.; Santato, C., Melanin: A Greener Route To Enhance Energy Storage under Solar Light. ACS Omega 2019, 4 (7), 1224412251.

(14) Kim, Y. J.; Wu, W.; Chun, S. E.; Whitacre, J. F.; Bettinger, C. J., Biologically Derived Melanin Electrodes in Aqueous Sodium-Ion Energy Storage Devices. Proceedings of the National Academy of Sciences 2013, 110 (52), 20912-20917.

(15) Felix, C. C.; Hyde, J. S.; Sarna, T.; Sealy, R. C., Interactions of Melanin with Metal Ions. Electron Spin Resonance Evidence for Chelate Complexes of Metal Ions with Free Radicals. Journal of the American Chemical Society 1978, 100 (12), 3922- 
3926.

(16) Panzella, L.; Gentile, G.; D’Errico, G.; Della Vecchia, N. F.; Errico, M. E.;

Napolitano, A.; Carfagna, C.; D'Ischia, M., Atypical Structural and $\pi-E l e c t r o n$

Features of a Melanin Polymer That Lead to Superior Free-Radical-Scavenging

Properties. Angewandte Chemie - International Edition 2013, 52 (48), 1-5.

(17) Chen, C. T.; Chuang, C.; Cao, J.; Ball, V.; Ruch, D.; Buehler, M. J., Excitonic Effects from Geometric Order and Disorder Explain Broadband Optical Absorption in Eumelanin. Nature Communications 2014, 5, 1-10.

(18) Büngeler, A.; Hämisch, B.; Huber, K.; Bremser, W.; Strube, O. I., Insight into the Final Step of the Supramolecular Buildup of Eumelanin. Langmuir 2017, 33 (27), $6895-6901$.

(19) McGinness, J.; Corry, P.; Proctor, P., Amorphous Semiconductor Switching in Melanins. Science 1974, 183 (4127), 853-855.

(20) Fritzsche, H., Switching and Memory in Amorphous Semiconductors, Ch. 6. In Amorphous and Liquid Semiconductors; Tauc, J., Ed.; Plenum Press: London and New York, 1974; pp 313-355. 
(21) Longuet-Higgins, H. C., On the Origin of the Free Radical Property of Melanins. Archives of Biochemistry and Biophysics 1960, 86, 231-232.

(22) Tauc, J., Optical Properties of Amorphous Semiconductors, Ch. 4. In Amorphous and Liquid Semiconductors; Tauc, J., Ed.; Plenum Press: London and New York, 1974; pp 159-214.

(23) Powell, M. R.; Rosenberg, B., The Nature of the Charge Carriers in Solvated Biomacromolecules. Bioenergetics 1970, 1 (6), 493-509.

(24) Di Mauro, E.; Carpentier, O.; Yáñez Sánchez, S. I.; Ignoumba Ignoumba, N.; Lalancette-Jean, M.; Lefebvre, J.; Zhang, S.; Graeff, C. F. O.; Cicoira, F.; Santato, C., Resistive Switching Controlled by the Hydration Level in Thin Films of the Biopigment Eumelanin. Journal Material Chemistry C 2016, 4 (40), 9544-9553.

(25) Wünsche, J.; Deng, Y.; Kumar, P.; Di Mauro, E.; Josberger, E.; Sayago, J.; Pezzella, A.; Soavi, F.; Cicoira, F.; Rolandi, M.; Santato, C., Protonic and Electronic Transport in Hydrated Thin Films of the Pigment Eumelanin. Chemistry of Materials 2015, $27(2), 436-442$.

(26) Wünsche, J.; Cicoira, F.; Graeff, C. F. O.; Santato, C., Eumelanin Thin Films: 
Solution-Processing, Growth, and Charge Transport Properties. Journal Material Chemistry B 2013, 1 (31), 3836-3842.

(27) Mostert, A. B.; Powell, B. J.; Pratt, F. L.; Hanson, G. R.; Sarna, T.; Gentle, I. R.; Meredith, P., Role of Semiconductivity and Ion Transport in the Electrical Conduction of Melanin. Proceedings of the National Academy of Sciences 2012, 109 (23), 8943-8947.

(28) Sheliakina, M.; Mostert, A. B.; Meredith, P., Decoupling Ionic and Electronic Currents in Melanin. Advanced Functional Materials 2018, 1805514.

(29) Sheliakina, M., Mostert, A.B., Meredith, P., An All-Solid-State Biocompatible Ion-toElectron Transducer for Bioelectronics. Materials Horizons 2018, 5(2), 256-263.

(30) Salleo, A., Electronic Traps in Organic Semiconductors, Ch. 14. In Organic Electronics: Emerging Concepts and Technologies; Cicoira, F., Santato, C., Eds.; Wiley-VCH, 2013; pp 341-373.

(31) Jastrzebska, M.; Kocot, A.; Tajber, L., Photoconductivity of Synthetic Dopa-Melanin Polymer. Journal of Photochemistry and Photobiology B: Biology 2002, 66, 201206. 
(32) Haneef, H. F.; Zeidell, A. M.; Jurchescu, O. D. Charge Carrier Traps in Organic Semiconductors: A Review on the Underlying Physics and Impact on Electronic Devices. Journal Materials Chemistry C 2020, 8(3), 759-787.

(33) Lin, W. P., Liu, S. J., Gong, T., Zhao, Q. , Huang, W., Polymer-Based Resistive Memory Materials and Devices. Advanced Materials 2014, 26, 570-606.

(34) Spólnik, P.; Król, M.; Stopa, B.; Konieczny, L.; Piekarska, B.; Rybarska, J.; Zemanek, G.; Jagusiak, A.; Piwowar, P.; Szoniec, G.; Roterman, I., Influence of the Electric Field on Supramolecular Structure and Properties of Amyloid-Specific Reagent Congo Red. European Biophysics Journa/2011, 40(10), 1187-1196.

(35) Huggins, R. A., Simple Method to Determine Electronic and Ionic Components of the Conductivity in Mixed Conductors. A Review. Ionics (Kiel) 2002, 8, 300-313.

(36) Jiang, T., Hall, A., Eres, M., Hemmatian, Z., Qiao, B., Zhou, Y., Ruan, Z., Couse, A. D., Heller, W. T., Huang, H., Olvera de la Cruz, M., Rolandi, M., Xu, T., SingleChain Heteropolymers Transport Protons Selectively and Rapidly. Nature 2020, 577, 216.

(37) Ferrari, A. C., Raman Spectroscopy of Graphene and Graphite: Disorder, Electron- 
Phonon Coupling, Doping and Nonadiabatic Effects. Solid State Communications

$2007,43,47-57$.

(38) Saenger, K. L.; Tsang, J. C.; Bol, A. A.; Chu, J. O.; Grill, A.; Lavoie, C., In Situ X-

Ray Diffraction Study of Graphitic Carbon Formed during Heating and Cooling of

Amorphous-C/Ni Bilayers. Applied Physics Letters 2010, 96, 2010-2013.

(39) Ambrico, M.; Ambrico, P. F.; Cardone, A.; Ligonzo, T.; Cicco, S. R.; Mundo, R. Di;

Augelli, V.; Farinola, G. M., Melanin Layer on Silicon: An Attractive Structure for a

Possible Exploitation in Bio-Polymer Based Metal-Insulator-Silicon Devices.

Advanced Materials 2011, 23 (29), 3332-3336.

(40) Ambrico, M.; Cardone, A.; Ligonzo, T.; Augelli, V.; Ambrico, P. F.; Cicco, S.;

Farinola, G. M.; Filannino, M.; Perna, G.; Capozzi, V., Hysteresis-Type Current-

Voltage Characteristics in Au/Eumelanin/ITO/Glass Structure: Towards Melanin

Based Memory Devices. Organic Electronics 2010, 11, 1809-1814. 


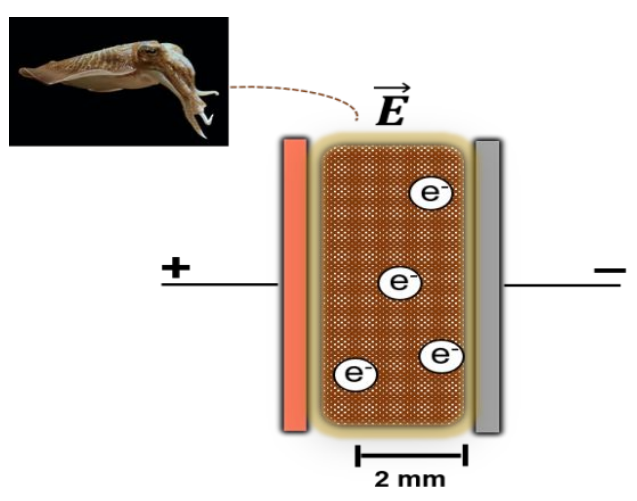

Table of content (ToC) graphic. 


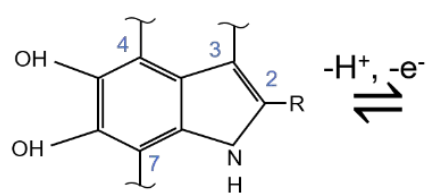

Hydroquinone (H2Q)

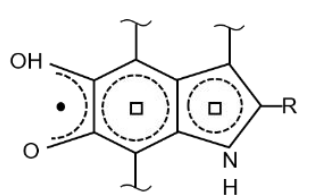

Semiquinone (SQ)<smiles>[R]c1[nH]c2c(c1C)=C(C)C(=O)C(=O)C=2C</smiles>

Quinone (Q)

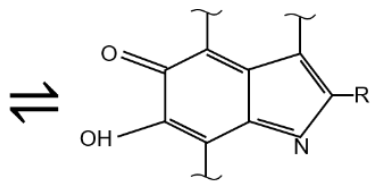

Quinone Imine (QI)

Scheme 1. Hydroquinone $(H 2 Q)$, semiquinone $(S Q)$ and quinone $(Q)$ redox forms of the building blocks of eumelanin: 5,6-dihydroxyindole ( $\mathrm{DHI})$ and 5,6-dihydroxyindole-2carboxylic acid (DHICA). $\mathrm{R}$ is $-\mathrm{H}$ in $\mathrm{DHI}$ whereas $\mathrm{R}$ is the $-\mathrm{COOH}$ group in DHICA. The quinone imine form (QI) is the tautomer of $Q$.<smiles>[Z10]C=CC=CC</smiles>

Scheme 2. The comproportionation equilibrium that regulates the relative concentrations of hydroquinone $(\mathrm{H} 2 \mathrm{Q})$, semiquinone $(\mathrm{SQ})$ and quinone $(\mathrm{Q})$ redox forms of the building blocks of eumelanin in the presence of water. Specifically, $\mathrm{H} 2 \mathrm{Q}$ and $\mathrm{Q}$ react to form protons and $S Q$ free radicals. 
A

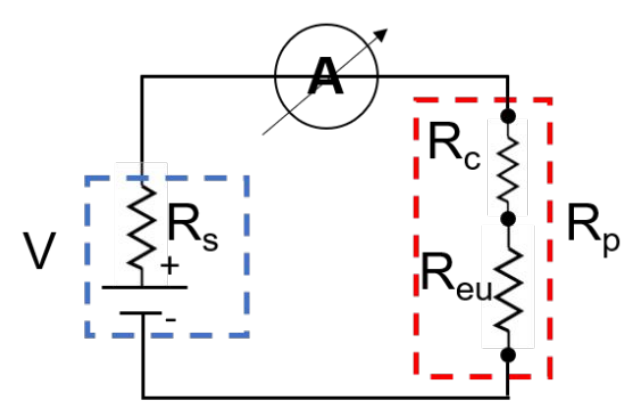

B

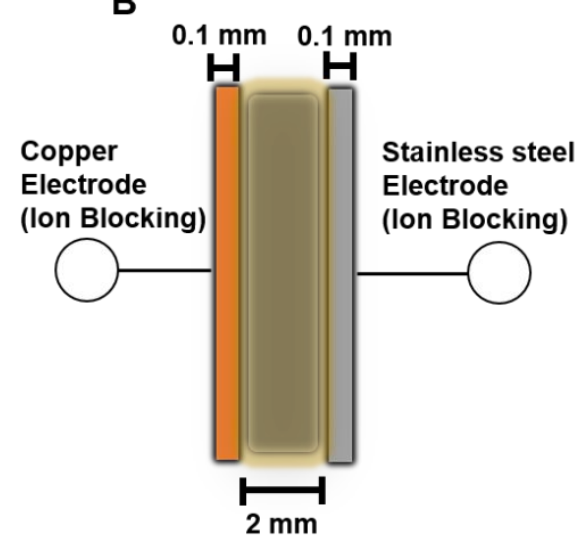

C

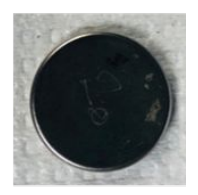

Scheme 3. Schematic representation of (A) the electrical circuit used in this work to acquire the electrical response of the pellets (B) the sandwich configuration of our Sepia melanin pellets (C) top view of Sepia melanin powders in a half coin cell. In (A) V is the voltage source, $\mathrm{A}$ is the amperometer in series with the resistance of the voltage source $\mathrm{R}_{\mathrm{s}}$ and the pellet resistance $\mathrm{R}_{\mathrm{p}}=$ $R_{e u}+R_{c}$, where $R_{e u}$ and $R_{c}$ are the intrinsic eumelanin resistance and the contact resistance respectively. 

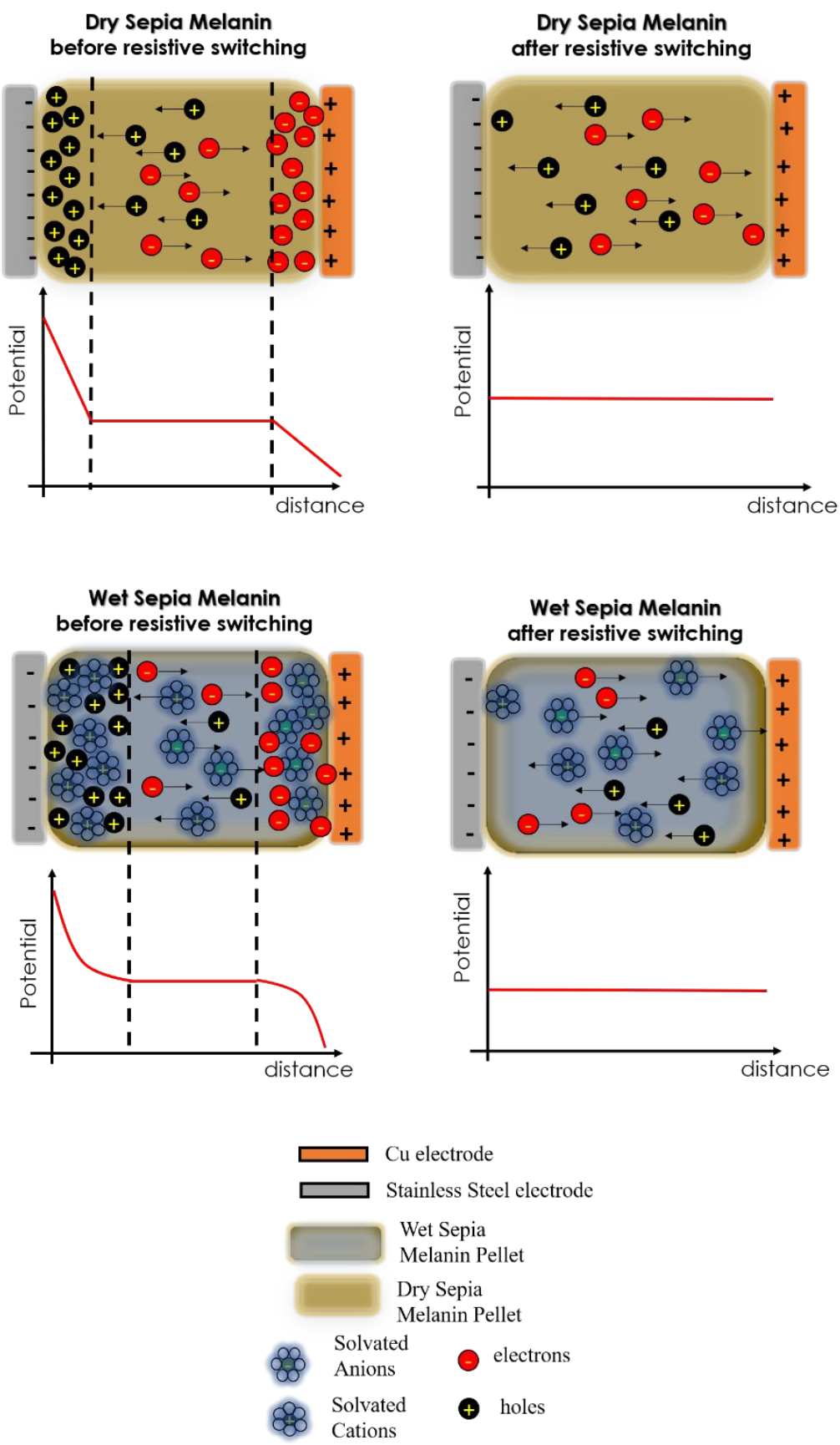

Scheme 4. Tentative model to describe the phenomenon of electrical resistive switching observed in dry and wet pellets of Sepia melanin, reported in this work. A key role in the 
model is played by the formation of electrical double layers (predominantly electronic when the pellets are dry and predominantly ionic when the pellets are wet) at the Sepia melanin /metal interfaces.
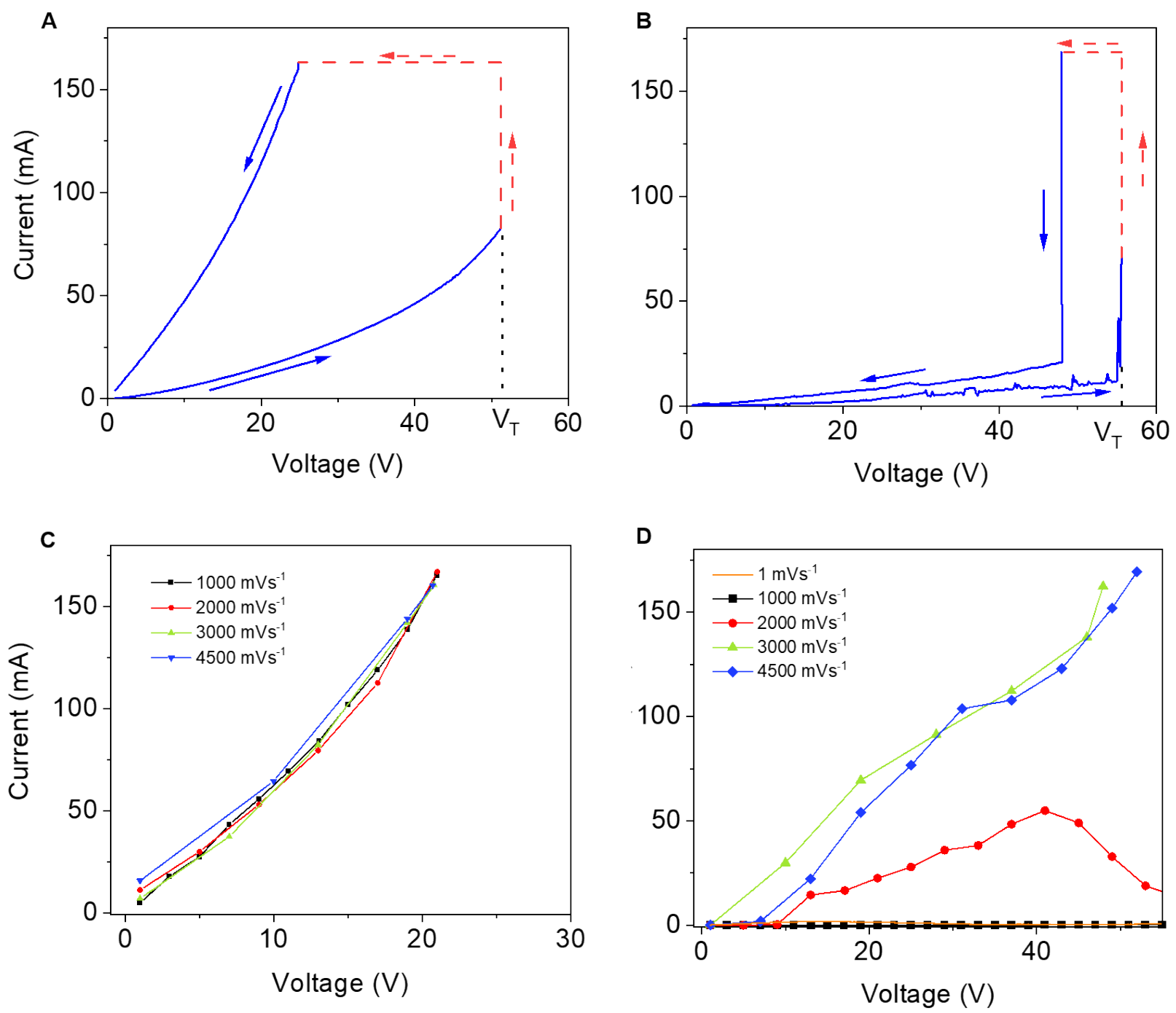

Figure 1. Current-voltage response at $100 \mathrm{mVs}^{-1}$ for $(\mathrm{A})$ dry and (B) wet pellets; sequential current-voltage acquisitions from low to high scan rates for (C) dry and (D) wet 
pellets (beyond $2000 \mathrm{mVs}^{-1}$, see also Figure S2). When the voltage source increases, the current monotonically increases up to the threshold voltage $\left(\mathrm{V}_{\mathrm{T}}\right)$. At $\mathrm{V}_{\mathrm{T}}$, because of the switching, the resistance of the pellet drops and the current increases non-monotonically up to the maximum current (maximum value of the current tolerated by the amperometer (Scheme 3A). Thus, from the portion of the plot where the source voltage increases to where the source voltage decreases a transition takes place. Since this transition is fast, only its start and end points can be measured. The real transition should appear as the contribution of a first line at constant voltage and of a second line at the constant maximum current (dash red lines and arrows). 


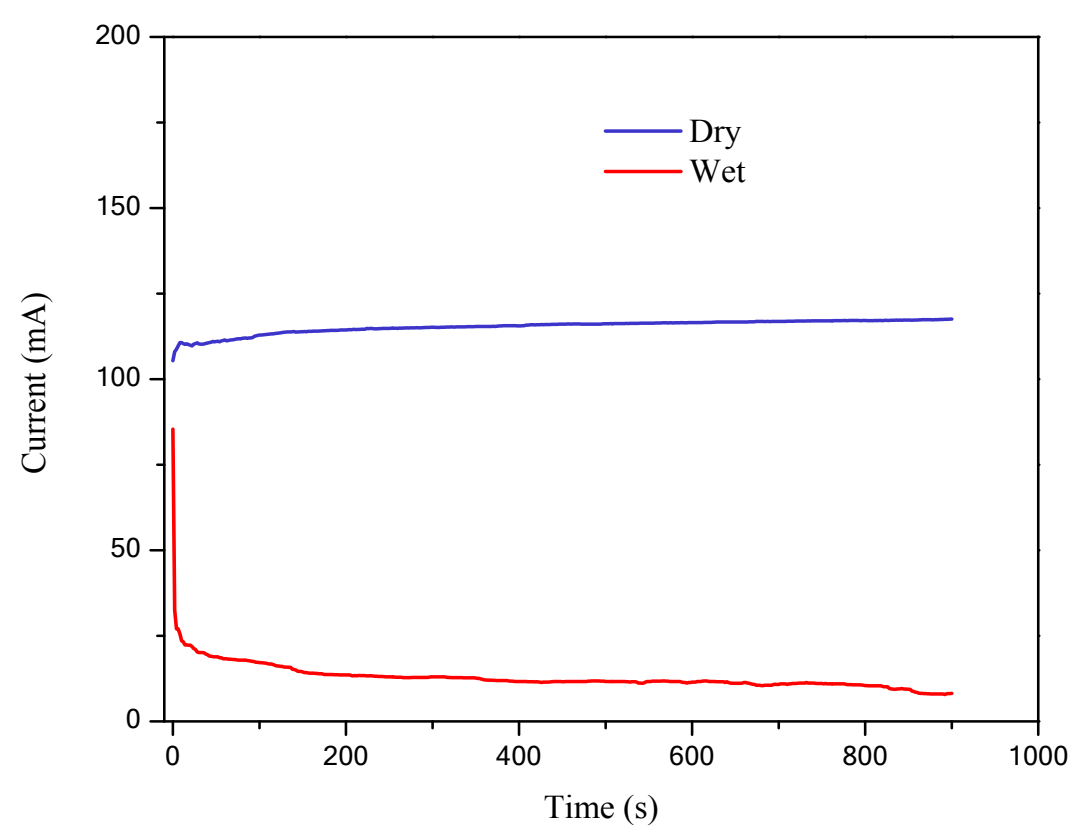

Figure 2. Current-time characteristics of wet and dry Sepia melanin pellets after resistive switching for dry ( $\mathrm{V}$ is about $25 \mathrm{~V}$, close to $\mathrm{V}_{\mathrm{T}}$ ) and wet Sepia melanin pellets ( $\mathrm{V}$ is about $45 \mathrm{~V}$, close to $\mathrm{V}_{\mathrm{T}}$ ). 

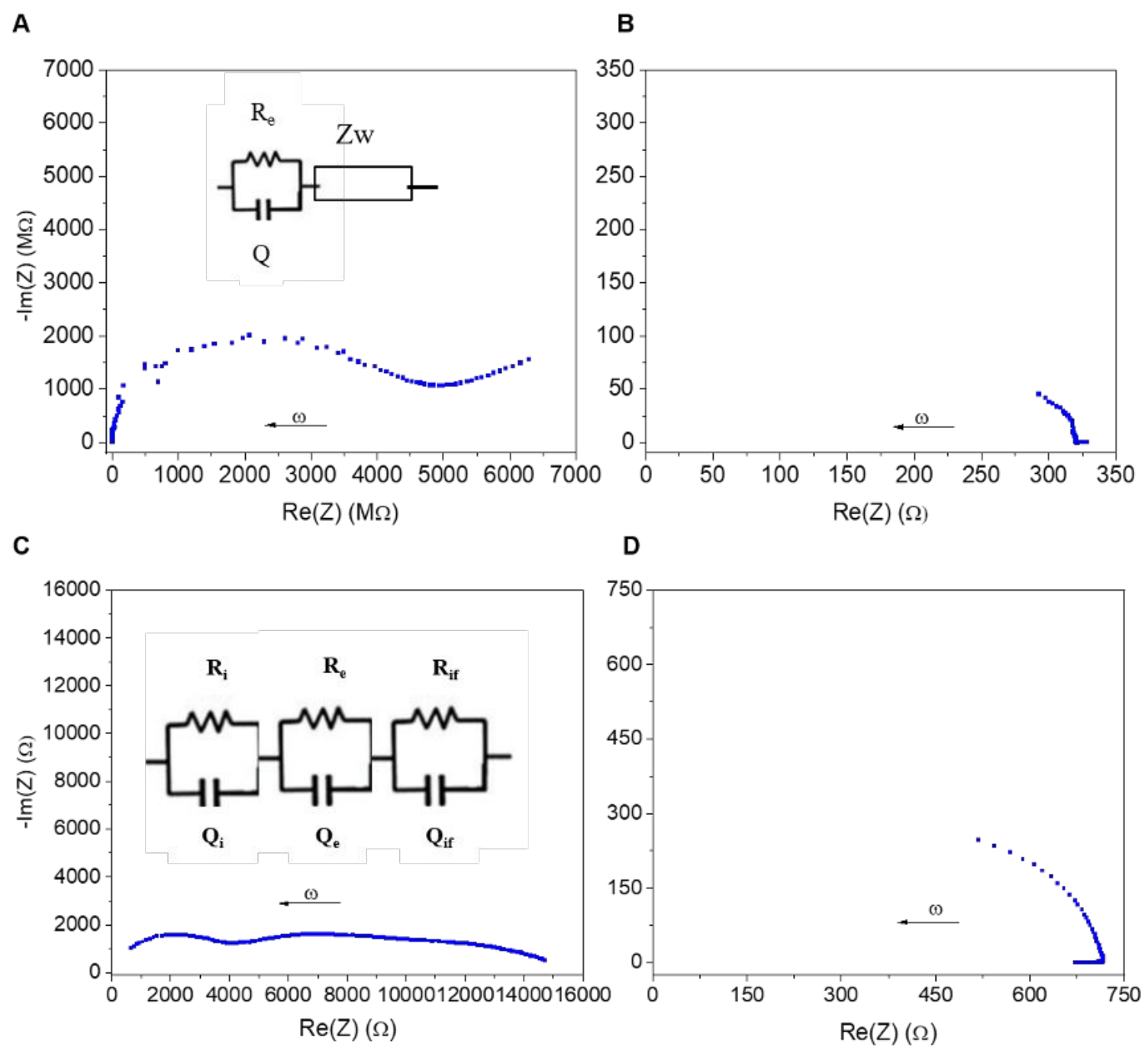

Figure 3. Electrochemical impedance spectroscopy of dry and wet Sepia melanin pellets.

Nyquist plots of dry, $(A)$ and $(B)$, and wet, $(C)$ and $(D)$, pellets; $(A)$ and $(C)$ before and $(B)$ and (D) after resistive switching. Insets of figures (A) and (C) corresponding to equivalent circuits where $R_{e}$ is the electronic resistance, $R_{i}$ the ionic resistance, $R_{i f}$ the interface resistance, $Q$ is the constant phase element (the series combination of melanin's 
geometrical capacitance and the capacitance at melanin/metal interface that describes

any deviation from an ideal capacitive response) and $Z_{w}$ the diffusion hindered impedance.

\begin{tabular}{|c|c|c|c|c|c|c|}
\hline Ref. & $\begin{array}{c}\text { Type of eumelanin } \\
\text { (form factor, dry versus } \\
\text { wet) }\end{array}$ & $\begin{array}{c}\text { Metal } \\
\text { contact }\end{array}$ & $\begin{array}{c}\text { Ion } \\
\text { content }\end{array}$ & $\begin{array}{l}\text { Interelectrode } \\
\text { distance }\end{array}$ & $\begin{array}{l}\text { Applied } \\
\text { Voltage }\end{array}$ & $\begin{array}{c}\text { Charge Carrier } \\
\text { Transport } \\
\text { Properties }\end{array}$ \\
\hline 19 & $\begin{array}{c}\text { Enzymatic action on } \\
\text { tyrosinase, autooxidation } \\
\text { of L-dopa, extracted from } \\
\text { melanoma tumor material, } \\
\text { homogenized tumor } \\
\text { (pellets, wet) }\end{array}$ & $\mathrm{Cu}, \mathrm{C}, \mathrm{Al}$ & - & $0.1,1,10 \mathrm{~mm}$ & $\begin{array}{l}\text { Interval } \\
0 \mathrm{~V}- \\
400 \mathrm{~V}\end{array}$ & $\begin{array}{c}\text { Electronic } \\
\text { Conductor } \\
\text { with Local } \\
\text { Modification of } \\
\text { the Dielectric } \\
\text { Constant }\end{array}$ \\
\hline 27 & $\begin{array}{l}\text { From solutions of DL- } \\
\text { dopa oxidized in air } \\
\text { (pellet, } 7-16 \% \text { water } \\
\text { content) }\end{array}$ & $\mathrm{Au}$ & - & $2 \mathrm{~mm}$ & $15 \mathrm{~V}$ & $\begin{array}{l}\text { Mixed lonic- } \\
\text { Electronic } \\
\text { Conductor }\end{array}$ \\
\hline 29 & $\begin{array}{l}\text { From solutions of DL- } \\
\text { dopa oxidized in air (film } \\
\text { used as ion gating } \\
\text { medium, wet) })^{1}\end{array}$ & $\mathrm{Au}$ & - & - & - & $\begin{array}{c}\text { Predominantly } \\
\text { Ionic } \\
\text { Conductor }\end{array}$ \\
\hline 39 & $\begin{array}{l}\text { Sigma melanin (film, } 40 \% \\
\text { RH in air and vacuum) }\end{array}$ & $\begin{array}{c}\mathrm{p}-\text { or } \mathrm{n}-\mathrm{Si} \\
\text { and } \mathrm{Au}\end{array}$ & - & $50 \mathrm{~nm}$ (diode) & ca $3 \mathrm{~V}$ & $\begin{array}{c}\text { Predominantly } \\
\text { Electronic } \\
\text { Conductor }\end{array}$ \\
\hline 40 & $\begin{array}{l}\text { Sigma melanin (film, } \\
\text { ambient conditions and } \\
\text { vacuum) }\end{array}$ & $\begin{array}{l}\text { ITO and } \\
\mathrm{Au}\end{array}$ & - & $400 \mathrm{~nm}$ (diode) & ca $1.5 \mathrm{~V}$ & $\begin{array}{c}\text { Predominantly } \\
\text { Electronic } \\
\text { Conductor }\end{array}$ \\
\hline 26 & $\begin{array}{l}\text { Sigma melanin (film, } \\
\text { interval included between } \\
50 \% \text { and } 90 \% \mathrm{RH} \text { ) }\end{array}$ & $\mathrm{Pt}$ & - & $\begin{array}{c}10 \mu \mathrm{m} \\
\text { (planar } \\
\text { configuration) }\end{array}$ & ca $1 \mathrm{~V}$ & $\begin{array}{c}\text { Predominantly } \\
\text { Ionic } \\
\text { Conductor }\end{array}$ \\
\hline
\end{tabular}




\begin{tabular}{|c|c|c|c|c|c|c|}
\hline 25 & $\begin{array}{c}\text { Sigma melanin obtained } \\
\text { by oxidation of tyrosine by } \\
\mathrm{H}_{2} \mathrm{O}_{2} \text { (film, } 12 \% \text { and } 17 \% \\
\text { water content) }\end{array}$ & $\begin{array}{c}\mathrm{Pt}, \mathrm{Pd}, \\
\mathrm{PdH}\end{array}$ & - & $\begin{array}{c}10 \mu \mathrm{m} \\
\text { (planar } \\
\text { configuration) }\end{array}$ & ca $1 \mathrm{~V}$ & $\begin{array}{c}\text { Predominantly } \\
\text { Ionic } \\
\text { Conductor }\end{array}$ \\
\hline 24 & $\begin{array}{l}\text { Sigma melanin and Sepia } \\
\text { melanin purchased from } \\
\text { Sigma (film, 90\% RH) }\end{array}$ & $\mathrm{Au}$ & $\begin{array}{c}\mathrm{Cl} \mathrm{ca} 7.0 \\
\mathrm{mg} / \mathrm{g} \\
\mathrm{Na} \mathrm{ca} 64 \\
\mathrm{mg} / \mathrm{g}\end{array}$ & $\begin{array}{c}6 \text { or } 10 \mu \mathrm{m} \\
\quad \text { (planar } \\
\text { configuration) }\end{array}$ & ca $1 \mathrm{~V}$ & $\begin{array}{c}\text { Ionic } \\
\text { Conductor } \\
\text { with Formation } \\
\text { of Metallic } \\
\text { Filaments }\end{array}$ \\
\hline $\begin{array}{c}\text { This } \\
\text { work }\end{array}$ & $\begin{array}{l}\text { Purified natural Sepia } \\
\text { melanin (pellet, dry and } \\
14 \% \text { and } 19 \% \text { water } \\
\text { content) }\end{array}$ & $\begin{array}{c}\mathrm{Cu} \text { and } \\
\text { stainless } \\
\text { steel }\end{array}$ & $\begin{array}{c}\mathrm{Cl} \mathrm{ca} \\
0.87 \\
\mathrm{mg} / \mathrm{g} \\
\mathrm{Na} \mathrm{ca} \\
3.9 \mathrm{mg} / \mathrm{g}\end{array}$ & $2 \mathrm{~mm}$ & $\begin{array}{c}\text { Interval } \\
0 \mathrm{~V}- \\
160 \mathrm{~V}\end{array}$ & $\begin{array}{c}\text { Dry Pellets: } \\
\text { Electronic } \\
\text { Conductors } \\
\text { Wet Pellets: } \\
\text { Ionic and } \\
\text { Electronic } \\
\text { Conductors }\end{array}$ \\
\hline
\end{tabular}

\section{Table 1. Literature on the charge carrier transport properties of eumelanin in the form of} wet and dry pellets and thin films. 